\title{
ACCELERATION AND PARTICLE FIELD INTERACTIONS OF COSMIC RAYS II: CALCULATIONS
}

\author{
${ }^{1,2}$ Tawfik, A., ${ }^{1}$ A. Saleh, ${ }^{3}$ M.T. Ghoneim and ${ }^{4}$ A.A. Hady \\ ${ }^{1}$ Egyptian Center for Theoretical Physics, Al-Mukattam, MTI University, Cairo, Egypt \\ ${ }^{2}$ Research Center for Einstein Physics, Freie-University Berlin, Berlin, Germany \\ ${ }^{3}$ Department of Physics, Faculty of Science, Cairo University, Cairo, Egypt \\ ${ }^{4}$ Department of Astronomy, Space Science and Meteorology, Faculty of Science, Cairo University, Cairo, Egypt
}

Received 2012-05-01, Revised 2012-06-12; Accepted 2013-07-17

\begin{abstract}
The Ultra High Energy Cosmic Rays (UHECRs) is still a one of the most controversial physical problems, especially that all potential acceleration mechanisms are not entirely able to explain the source of UHECR and their energy scale. Based on our generic acceleration model, that suggests different types of electromagnetic interactions between the cosmic charged particles and the different configurations of the electromagnetic (plasma) fields, UHECR are systematically analyzed. The plasma fields are assumed to vary, either spatially or temporally or both. In this approach, the well-known Fermi accelerations are excluded. But seeking for simplicity, it is assumed that the energy loss due to the different physical processes is negligibly small. The energy available to the plasma sector is calculated in four types of electromagnetic fields. It is found that the drift in a time-varying magnetic field is extremely energetic and the energy scale widely exceeds the Greisen-Zatsepin-Kuzmin (GZK) cutoff. We deduced that the polarization drifts in a time-varying electric field is also able to raise the energy of UHECR to an extreme value. Thus, this type of generic mechanisms seems to be combatiple with the Hillas mechanism. The drift in a spatially-varying magnetic field is almost as strong as the polarization drift. The curvature drift in a non-uniform magnetic field and a vanishing electric field is very weak. It is to be concluded that the studying cosmic charged particle interactions with the different aspects of the cosmic plasmas could be considered as intrinsic sources for UHECR and therefore a remarkable portion of the ultra high energies that are observed in the cosmic rays can be interpreted.
\end{abstract}

Keywords: Greisen-Zatsepin-Kuzmin (GZK), UHECR, Electromagnetic Inter-Actions

\section{INTRODUCTION}

The Fermi acceleration mechanisms (Fermi, 1949; Blandford and Eichler, 1987) have been extended to include different types of electromagnetic (plasma) interactions (Tawfik et al., 2012). In present study, the total energy gained by cosmic charged particles is calculated according the generic acceleration model (Tawfik et al., 2012). The arguments given in Ref. Tawfik et al. (2012) on introducing non-relativistic formalism, are respected in the present study. It is aimed to show that the plasma field interactions, which originally are not implemented in the Fermi mechanisms (first- and second-order), can contribute with a considerable amount to the Ultra High Energy of Cosmic Rays (UHECR). In the generic acceleration model (Tawfik et al., 2012), the astrophysical objects, in which one or more types of plasma fields have been observed and/or predicted, are taken into consideration. On the other hand, the energy loss due to the different physical processes such as synchrotron, curvature and Bremsstrahlung radiation is entirely disregarded. The

(c) 2013 Tawfik, A., A. Saleh, M.T. Ghoneim and A.A. Hady. This open access article is distributed under a Creative Commons Attribution (CC-BY) 3.0 license which permits unrestricted use, distribution, and reproduction in any medium, provided the original work is properly credited. DOI: 10.3844/pisp.2013.29.36 
electric potential difference and $E \times B$, polarization, curvature and gravitational drifts are conjectured as sources for accelerating the cosmic charged particles by plasma field interactions. The plasma fields themselves can be homogeneous, non-homogeneous or timespatially varying. It is obvious that the numerical calculations offer a tool to judge about the most effective mechanism, which likely derives the cosmic charged articles to an extreme energy value.

The origin of UHECR is one of the greatest mysteries in modern physics. In order to compare their energy scale, let us recall that the current acceleration technologies are merely able to come up with $\mathrm{O}\left(10^{12}\right) \mathrm{eV}$. On the other hand, several observatories around the world have registered cosmic rays with primary energies $>10^{20} \mathrm{eV}$ (Collaboration et al., 2007; Abraham et al., 2009; Yoshida and Dai, 1998; Nagano and Watson, 2000; Takeda et al., 1998; Teshima, 2001). From the theoretical point of view, the protons, which are the dominant components of UHECR, would posse's energy values much higher than the so-called Greisen-Zatsepiti-Kuzmin (GZK) cutoff $10^{19.7} \mathrm{eV}$. They are conjectured to suddenly lose their energies in photopion production processes with the cosmic microwave background radiation (Greisen, 1966; Zatsepin and Kuzmin, 1966; Nagano and Watson, 2000).

When a charged particle moves with reference to an equipotential surface where the electric potential $\phi$ is constant and the existing electric field is stationary, then the equipotential surface is considered not to be coincide with themagnetic surface. In other words, the components of electric $\mathrm{E}$ and magnetic field strength $\mathrm{B}$ are perpendicular to each other. This leads to an $E \times B$ drift with velocity $\mathrm{V} \perp$ and an energy $\varepsilon=\mathrm{q} E \cdot r$, where $\mathrm{q}$ is the static electric charge and $r$ is the distance covered by the accelerated particle. This idea has been proposed by (Hillas, 1984) long time ago. In the generic acceleration model, this basic idea has been rather generalized. The configurations of the electromagnetic fields are designed to cover all possibilities, including the one in which the equipotential surface coincides with the magnetic surface.

According to the generic model, the energy gained by cosmic charged particles along their path through a decreasing electric potential in a totally conserved field with varying spatial intensities is given as $\varepsilon=2 \mathrm{qE} \cdot \mathrm{r}$. That this model predicts two times the Hillas energy can be understood according to the configuration of the fields and their variation in spatial and/or temporal dimensions.

It is conjectured that the magnetic fields of the cosmic plasmas likely verify the so-called frozen-in condition which means that the magnetic flux $\Psi=\mathrm{B} \mathrm{R}^{2}$ is globally conserved (Contopoulos et al., 1999). Both electric and magnetic fields are correlated. They move coincidentally. On the other hand, the ideal MagnetoHydro-Dynamic (MHD) plasmas is conjectured to satisfy the condition $\mathrm{E} \cdot \mathrm{B}=0$ everywhere. They ensure that the generalized Ohm's law $\mathrm{E}=-\mathrm{V} \times \mathrm{B}$. The well-known Hillas criterion essentially relies on both conditions and leads to the non-relativistic relation $\varepsilon=\mathrm{q} V \mathrm{~B} \mathrm{R}$, where $\mathrm{R}$ is the characteristic size of linear accelerator and $\mathrm{V}$ is the velocity of the background plasmas. Hillas graph orders the magnetic field strength $\mathrm{B}$ versus the linear size $\mathrm{R}$ for various astrophysical objects. It is found that the total energy, which would be available to the cosmic charged particles from their interactions with the magnetic fields described in Hillas model, can be as large as $10^{20} \mathrm{eV}$ (Gopalswamy et al., 2010). In the generic model (Tawfik et al., 2012), the configurations of $\mathrm{E}$ and $\mathrm{B}$ are generalized. This results in two components. First one is suppose to be responsible for the gyration perpendicular to both electric and magnetic fields. The other one is the accelerating component. Directions of both components, acceleration and gyration are coincident (Tawfik et al., 2012). In present study, we introduce numerical calculations for the energy gained in these fields.

Alternative hypotheses about the acceleration through electrostatic potential difference have been modelled for var-ious astrophysical objects (Becker, 2009). Neutron stars (like pulsars, magnetars) are well known examples of astrophysical objects, in which electrostatic potential might be available. From theoretical point of view, standard pulsar magne- tospheric, polar cap and slot gap models have been utilized (Becker, 2009) in order to explain the ultra high energy based on electrostatic acceleration. Accordingly, the electric potential difference between center and edge of the polar cap is given as $\Delta \phi \approx \Omega B_{s} r^{2} / 2 c$, where $r$ is the radius, is the angular velocity and $B_{s}$ stands for magnetic field strength at the surface. Such a result can also be implemented in the generic model. The correlations between coordinates of UHECR and stellar objects can be taken into consideration as well. This would offer an alternative method to find out additional sources for UHECR and would be a next extension to the generic acceleration model.

The present study is organized as follows. The energy gained by cosmic charged particles in various electromagnetic fields is calculated. Examples for the drift energies arising in four types of electromagnetic fields are elaborated as follows. The drift of spatially varying magnetic is devoted to the curvature drift arising 
in a non-uniform magnetic field and a vanishing electric field. The polarization drift of the time-varying electric field is. Last but not least, the time-varying magnetic field has been utilized to calculate the difference of drift energy. The discussion and final conclusions are given. This might explain the extreme high energy that the cosmic charged particles would gains in these types of electromagnetic fields.

\subsection{Drift Energies in Various Electromagnetic Fields}

We calculate the energy that the cosmic charged particles would gain through their interactions with the different types of the plasma fields (Tawfik et al., 2012). As discussed in the introduction, the generic acceleration model offers a complete set of extensions to Hillas idea, where ExB drifts have been calculated, exclusively. It is worthwhile to remember again, that these field interactions would come up with a considerable part, may be a very large portion, of the total energy. Fermi acceleration mechanisms, which have been intensively studied over the last few decades, contribute with another considerable part. The energy loss due to various physical processes would reduce the total energy with a non-negligible amount. Since these processes would be very dominant under very special conditions and certainly rely on a very rich physics, we therefore like to study them quantitatively in a future study. Seeking for simplicity, we assume that such physical processes are very weak to be neglected.

\subsection{Spatially Varying Magnetic Field B}

The gradient drift is associated with a nonhomogeneous magnetic field $\mathrm{B}$ and a vanishing electric field, $\mathrm{E}=0$.

We assume a very simple case of a spatially-varying magnetic field. A particle of mass $m$ and static electric charge $\mathrm{q}$ moving with velocity $\mathrm{v}$ in such magnetic field would be accelerated to an average energy:

$$
\langle\varepsilon\rangle=\mathrm{q} \frac{\mathrm{vr}_{1}}{2} \cdot \mathrm{r} \frac{\mathrm{dB}_{\mathrm{z}}}{\mathrm{dy}}
$$

Equation 1 shows the average energy gained by the cosmic charged particle due to the gradient of the magnetif field, where, the variation of this magnetic field is assumed to be in z-direction and Equation 2:

$$
\frac{\mathrm{dB}_{\mathrm{z}}}{\mathrm{dy}} \approx \frac{\mathrm{B}_{\mathrm{z}}}{\mathrm{L}} \square \frac{\mathrm{B}_{\mathrm{z}}}{\mathrm{r}_{1}}
$$

$\mathrm{L}$ is the characteristic size of the acceleration area. $\mathrm{r}_{1}$ is defined as the Larmor radius. In order to assure that the particle does not exceed the horizon of the acceleration area, the inequality given in Equatioin 2 has to be valid, i.e., $L$ has to be much greater than $r_{1}$. Let us assume that the cosmic charged particle gyrates along $\mathrm{Z}$ and y directions and covers a distance $r_{L} \approx L$. The last assumption is essential to assure that particle is about to leave the acceleration area. Thus:

$$
\langle\varepsilon\rangle \approx \mathrm{q} \frac{\mathrm{vL}}{2} \mathrm{~B}_{0}
$$

where, $B_{0}$ is the surface magnetic field strength of the stellar object. In the scope of Equation 3 the cosmic charged particle could achieve the maximum average energy, as the Larmor radius $r_{1}$ approaches to the characteristic size of the acceleration environment $\mathrm{L}$.

To answer the question about the nature of stellar objects, in which such a non-uniform magnetic field would be possible, we suggest the neutron stars which inhibit a very strong magnetic field strength spatially ranging from $10^{9}-10^{12} \mathrm{G}$ depending on the radius or distance covered by the cosmic particle inside the magnetic field. Crab-pulsar and magnetars are well-known examples of stellar objects with extreme magnetic fields. The time period of their spinning rotation apparently affect the strength of the magnetic fields. Younger neutron stars would have a very much strong magnetic field and even a shorter time period of spinning $(33 \mathrm{~ms})$ than the elder ones (Ghisellini et al., 2008). The structure formation shocks (Inoue, 2008) are conjectured as possible sources. Also, spinning black holes have been suggested (PrescodWeinstein et al., 2009) to accelerate the cosmic charged particle to ultra high energies, Table 1.

Another method to estimate an arbitrary Larmor radius $r_{1}$ would be its relation with the magnetic field strength B (Tawfik et al., 2012). Then, the energy drift in non-uniform magnetic fields of neutron stars would be as much as $\sim 4.9 \times 10^{16} \mathrm{eV}$, which is apparently too small to assure ultra high energy to the cosmic charged particles. In Table 1, we list the energies gained by cosmic charged particle in different astrophysical objects.

Such a result seems to verify Hillas model, which assumes that the energy gained by cosmic charged particles stems from a special type of drifts, explicitly. This is the polarization drift as will be given. The generic acceleration model (Tawfik et al., 2012) assumes that this energy is to be considered as one source. The Fermi accelerations and other types of field interactions are other sources. 
Table 1. Expansions and magnetic field strengths of different astrophysical objects are used to calculate the energy the the cosmic charged particles would gain from the plasma field interactions. In these calculations, the velocity $\mathrm{v}$ is assumed to be nonrelativistic; $\mathrm{v} \sim 0.1 \mathrm{c}$ and the cosmic charged particles are assumed to curry a unit charge, $\mathrm{q}=1.6 \cdot 10^{-19} \mathrm{C}$. The last line is added to compare with ordinary astrophysical objects with the Cen-A

\begin{tabular}{llll}
\hline Object & $\mathrm{R}(\mathrm{m})$ & $\mathrm{B}(\mathrm{Tesla})$ & $\varepsilon(\mathrm{eV})$ \\
\hline Neutron stars & $10^{4}$ & $10^{5}-10^{8}$ & $1.6 \cdot 10^{16}-1.6 \cdot 10^{19}$ \\
Magnetars & $10^{4}$ & $10^{8}-10^{11}$ & $1.6 \cdot 10^{19}-1.6 \cdot 10^{22}$ \\
Pulsars & $10^{4}$ & $10^{9}$ & $1.6 \cdot 10^{20}$ \\
Pulsar wind & $3 \cdot 10^{13}$ & $10^{-9}$ & $0.5 \cdot 10^{12}$ \\
SNR & $3 \cdot 10^{17}$ & $10^{-10}$ & $0.5 \cdot 10^{15}$ \\
Normal Stars & 108 & $10^{-7}-10^{-1}$ & $1.6 \cdot 10^{8}-1.6 \cdot 10^{14}$ \\
AGN & $10^{13}$ & $10^{-4}$ & $1.6 .0^{16}$ \\
AGN (Nucleus) & $3 \cdot 10^{12}$ & $10^{-1}$ & $0.5 \cdot 10^{19}$ \\
AGN (Related Jets) & $3 \cdot 10^{13}$ & $10^{-4}$ & $0.5 \cdot 10^{17}$ \\
AGN (Hot Spots) & $3 \cdot 10^{19}$ & $10^{-8}$ & $0.5 \cdot 10^{19}$ \\
AGN (Radio Lobes) & $3 \cdot 10^{22}$ & $10^{-10}$ & $0.5 \cdot 10^{20}$ \\
Galactic Disk & $10_{18}$ & $10^{-10}-10^{-9}$ & $1.6 \cdot 10^{15}-1.6 \cdot 10^{16}$ \\
Galactic Cluster & $3 \cdot 10^{23}$ & $10^{-11}$ & $0.5 \cdot 10^{20}$ \\
GRB & $3 \cdot 10^{11}$ & $10^{-1}$ & $0.5 \cdot 10^{18}$ \\
Cen-A & $2.5 \cdot 10^{20}$ & $10^{-9}$ & $0.4 \cdot 10^{19}$ \\
\hline
\end{tabular}

\subsection{Non-Uniform Magnetic Field $B$ and Vanishing Electric Field E (Curvature Drift)}

We assume that the charged particle moves parallel to the field lines. The latter are in turn assumed to be curved. Therefore, the particle experiences a centrifugal force. Under the assumption that the electric field $\mathrm{E}$ vanishes and the magnetic field $\mathrm{B}$ being timeindependent and weakly inhomogeneous, then the charged particle would gyrate around the magnetic field lines describing a helical path with Larmor radius $r_{1}$ (Tawfik et al., 2012). Under these conditions, we get:

$$
\mathrm{F}_{\mathrm{R}}=\mathrm{mv}_{\square}^{2} \frac{\mathrm{R}_{\mathrm{c}}}{\mathrm{R}_{\mathrm{c}}^{2}}
$$

where, $R_{c}$ is the local radius of curvature. Inserting the equation of general force drift Equation 5:

$\mathrm{v}_{\mathrm{d}}=\frac{1}{\mathrm{q}} \frac{\mathrm{F} \times \mathrm{B}}{\mathrm{B}^{2}}$

Into Equation 4 leads to an expression for the curvature drift:

$\mathrm{v}_{\mathrm{R}}=\frac{\mathrm{mv}_{\|}^{2}}{\mathrm{q}} \frac{\mathrm{R}_{\mathrm{c}} \times \mathrm{B}}{\mathrm{R}_{\mathrm{c}}^{2} \mathrm{~B}^{2}}$

It is obvious that $v_{R}$ is a constant velocity. According to Equation 6, the acceleration vanishes.
Therefore, the curvature drift-in non-relativistic limitseems to be proportional to the parallel component of the particle energy; $\varepsilon_{\square}=\frac{1}{2} \mathrm{mv}_{\square}^{2}$.

In relativistic limit, this component can be written as:

$\varepsilon_{\|}=\gamma \mathrm{m}_{\mathrm{o}} \mathrm{c}^{2}$

It is apparent, that the energy of Equation 7 does not depend on the astrophysical parameters such as electric field $\mathrm{E}$, magnetic field $\mathrm{B}$, expansion $\mathrm{r}$. The maximum energy value would be of the order of $\mathrm{O}\left(10^{6}\right) \mathrm{eV}$.

\subsection{Time-Varying Electric Field E (Polarization Drift)}

Assuming that the magnetic field strength $\mathrm{B}$ is homogeneous and non-varying while the electric field strength $\mathrm{E}$ is temporarily varying within the acceleration area. At a particular distance $r$, the acceleration and energy are given as follows:

$a=\frac{m}{q^{2}}\left(-\omega^{2} E(r, t)\right)$

$E(r, t)=E_{0} \exp (i \omega t)$

According to Equation 9 the electric field variation in this current case (time-varying electric field E) could describe an exponential variation, where, angular 
velocity $\omega$ is related to the radius $r_{L}$. Utilizing Ohm's law in MHD-plasma and $\mathrm{E}=-\mathrm{V} \times \mathrm{B}$, then the acceleration in Equation 8 can be rewritten as:

$$
\mathrm{a}=\mathrm{q} / \mathrm{m}(\mathrm{V} \times \mathrm{B})
$$

Equation 10 reveals that the acceleration of the cosmic charged particle depends on the configuration of the electromagnetic field. Here, the electric and magnetic fields are perpendicular to each other and hence, the acceleration direction will be in the perpendicular direction to the plane of $\mathrm{V}$ and $\mathrm{B}$.

Assuming a special case, in which both $\mathrm{V}$ and $\mathrm{B}$ are perpendicular to each other, then the polarization energy drift simply reads:

$$
\mathrm{e}=\mathrm{qVBR}
$$

where, $\mathrm{R}$ and $\mathrm{V}$ being the linear size of the astrophysical object and velocity of the background plasma, respectively. Equation 11 seems to remind with the Hillas energy. It is notable that the polarization drift of a charged particle looks similar to Hillas model. It is obvious that the latter is nothing but twice the energy gained by the same cosmic charged particle in a spatially varying B.

\subsection{Time-Varying Magnetic Field B (Grad E Drift)}

Assuming that the magnetic field B is not constant over the entire time interval $t$, then we can apply Faraday's law so that the time-varying magnetic field strength, dB/dt, turns to be given by $-\nabla \times E$. Such a relation apparently implies that the time-varying B generates a"grad E" drift. The latter raises the acceleration so that the change in the energy reads:

$$
\delta \mathrm{E}_{\mathrm{VE}}=2 \pi \frac{\mu}{\Omega_{\mathrm{c}}} \frac{\mathrm{dB}(\mathrm{t})}{\mathrm{dt}}
$$

where, $\mu$ is the magnetic moment. It is given by $\mathrm{mv}_{\perp}^{2} / 2 \mathrm{~B} . \Omega_{\mathrm{c}}$ is the cyclotron frequency, which is equivalent to $\mathrm{qB} / \mathrm{m}$. With respect to Equation 12 the time varying magnetic field is able to accelerate the cosmic particles, as the Faraday's law is satisfied. Therefore, an electric field is produced which is able to accelerate the cosmic particles to appreciable energies. It is obvious that this drift strongly depends on the particle's mass, $\mathrm{m}$ and magnetic field strength B Equation 13:

$$
\delta \mathrm{E}_{\mathrm{VE}}=\frac{\pi}{\mathrm{q}}\left(\frac{\mathrm{mv} \perp}{\mathrm{B}_{0}}\right)^{2} \frac{\mathrm{dB}(\mathrm{t})}{\mathrm{dt}}
$$

Again, the crab pulsars and/or neutron stars would be subject to this drift. In these astrophysical objects, phases of magnetic growth have been conjectured. Many authors (Woodward, 1984; Blandford et al., 1983) have suggested that the magnetic fields in neutron stars, for instance, can be explained by the so-called thermally driven battery mechanism. The latter means that the neutron starts would possess a magnetic field of $\leq 10^{8} \mathrm{G}$ at their birth and over a period of $\sim 10^{5}$ y this magnetic field raises to $\sim 10^{12} \mathrm{G}$. Other mechanisms which temporarily affect the magnetic field include the rotation period, matter transfer phase and periods of spin-up and down, especially, in the dipole fields. In the growth phase, the temporarily growing magnetic field strength $B$ (t) and spindown age $t_{s}(t)$, respectively, would read (Bhattacharya and Shukre, 1985):

$\mathrm{B}(\mathrm{t})=\mathrm{B}_{0} \exp (\mathrm{t} / \tau)$

$\mathrm{t}_{\mathrm{s}}(\mathrm{t})=\mathrm{t}_{\mathrm{s} 0} \exp (-2 \mathrm{t} / \tau)+\frac{\tau}{2}[1-\operatorname{EXP}(-2 \mathrm{~T} / \tau)]$

where, $\tau$ stands for the characteristic timescale for the growth of the magnetic field. Equation 14 and 15 describe an exponential growth. Regardless the GZKcutoff (Greisen, 1966; Zatsepin and Kuzmin, 1966), a simple calculation shows that this magnetic field would be able to accelerate the proton up to $\sim 10^{22} \mathrm{eV}$. This huge energy value would be available to protons which are positioned in this rapidly changing magnetic field and after the acceleration they will able to fly away from the accelerating stars. The well-known Fermi mechanisms are not taken into account. Also, the energy loss is not calculated.

Few remarks on the origin of magnetic fields in pulsars and magnetars are now in order. There are three scenarios, (1) generation from fossil fields, (2) internal generation in progenitor and (3) generation during core collapse (Spruit, 2008). The third scenario is very much interesting. Tidal flows and deformations have been suggested to explain the generation of magnetic fields in binary celestial systems. These processes are reversible in a short period. Also, merging of a binary neutron star system has been suggested to produce a extremely rapid mechanism for ultra-strong magnetic field (Price and Rosswog, 2006). This has been suggested to be responsible for the short class of Gamma Ray Bursts 
(GRB). MHD fields are amplified via Kelvin-Helmholtz instabilities beyond magnetar field strength. They might represent the strongest magnetic fields in the universe. The amplification seems to occur in the shear layer positioned between the binary stars and at a time scale of $\sim 1 \mu \mathrm{s}$. Therefore, the energy that a singly charged particle, like proton, would gain seems to be incredibly high.

\section{MATERIALS AND METHODS}

In the present work, we utilized models in order to estimate the potential acceleration of the cosmic charged, massive particles when passing through diiferent typles of plasma fields. The mathematical methods are basically based on applying fundamental rules given by electromagnetic interactions with interstellar objects. Enrico Fermi apparwently drawn the same line, couple decades ago. The materials available to this work are rich litrature, previous investigations and successful models. On top of this list, we put the observatiobal confirmation of the relation governing the cosmic ray energies.

\section{RESULTS AND DISCUSSION}

In this present work, we calculate the maximum achievable energy of the cosmic charged particle in various astrophysical plasma spects. Obviously, the maximum attainable energy due to the generic model seems to be twice the energy introduced by the Hillas argument, which depends on the $\mathrm{E} \times \mathrm{B}$ drift. In this regard, we deduced that the polarization drift can raise the energy of the cosmic charged particle to be UHECRs. Furthermore, polarization drift energy is equal to the acquired energy by the Hillas conditions. The spatially varying magnetic field $B$ introduces half energy suggested by $\mathrm{E} \times \mathrm{B}$ drift (Hillas argument). The non-uniform magnetic field $B$ and vanishing electric field $\mathrm{E}$ (Curvature drift) is too feeble to interpret the UHECRs, as it imparts the cosmic particles energy of order $10^{6} \mathrm{eV}$. The time-varying magnetic field B (grad E drift) could be a vibrant source for UHECRs. The magnetosphere of the pulsars and magnetars is a suitable astrophysical environment for this case. Therefore, the maximum attainable energy is up to $10^{22}$ $\mathrm{eV}$, where the energy loss due to different physical processes is entirely excluded. So that, the different aspects of electromagnetic fields through the plausible sources of UHECRs could be strongly considered in the acceleration process.

\section{CONCLUSION}

The energy that cosmic charged particles would gain from their interactions with different types of electromagnetic fields has been calculated. It has been found that the energy drifts can be as large as the GZK-cutoff (Greisen, 1966; Zatsepin and Kuzmin, 1966), although Fermi mechanisms are excluded. It is essential to mention here that the energy loss due to various physical processes has been disregarded, as well. They would be very dominant under very special conditions and certainly rely on a very rich physics. Seeking for simplicity, we assume that such the energy-reducing processes are very weak to be neglected. Nevertheless, a quantitative estimation will be elaborated in a future study. In present work, four types of plasma drifts have been calculated for a particle of mass $\mathrm{m}$ and static electric charge $\mathrm{q}$. The particle initial velocity, either finite or vanishing $\mathrm{v}$, shall be increased over its path through the plasma field. The utilized model (Tawfik et al., 2012) assumes general configurations for electric $\mathrm{E}$ and magnetic field strength B. Such an arrangement results in two components. The first one is responsible for the gyration perpendicular to both electric and magnetic fields. The other one is responsible for the acceleration. It has been found that the directions of both components, acceleration and gyration are coincident. The spatially varying magnetic field $B$ seems to verify Hillas model. The latter assumes that the energy gained by cosmic charged particles stems from a special type of drifts, explicitly. This is the polarization drift. The generic acceleration model (Tawfik et al., 2012) assumes that this energy is to be considered as one source. The Fermi accelerations and other types of field interactions are other sources.

The neutron stars, especially the younger ones, inhibit very strong magnetic fields, which seems to vary from $10^{9}-10^{12} \mathrm{G}$ depending on the radius or distance covered by the accelerated particle. The crab-pulsar and magnetars are stellar objects with extreme magnetic fields. The time period of their spinning rotation apparently affect the strength of magnetic fields. The spinning periods of younger neutron stars secure extreme magnetic fields. The structure formation shocks and spinning black holes can accelerate cosmic charged particle to ultra high energies.

The drift of a non-uniform magnetic field $\mathrm{B}$ and a vanishing electric field $\mathrm{E}$ seems to be very weak, so that the maximum energy is just couple $\mathrm{M} \mathrm{eV}$. Apparently, this kind of drifts is responsible for curving the 
accelerated particle around the non-uniform magnetic field B. Therefore, it would feed the final energy with a small amount. Even in the relativistic limit, the contribution of the curvature drift is minimum.

The drift in a time-varying electric field E gives an energy value, which is related to magnetic field strength. It reflects the Ohm's law and the configurations of the two fields ( $\mathrm{E}$ and $\mathrm{B}$ ). In MHD plasma, $\mathrm{E}$ and $\mathrm{B}$ are perpendicular to each other. Therefore, the relation between them is governed by the Ohm's law. As discussed above, this polarization drift results in the well-known Hillas energy scale. Last but not least, the drift in a time-varying magnetic field B, so-called"grad E" drift, seems to be very much effective. It relies on Faraday's law. On the other hand, the final energy that the cosmic charged particle would gain in this field is directly related to the time-varying magnetic field strength. Crab pulsars and neutron stars are conjectured to have phases, in which the magnetic field rapidly changes with the time. Accordingly, their fields have been explained by thermally driven battery mechanism. Rotation, matter transfer, spinup and -down periods, especially, in the dipole fields, are examples of additional mechanisms which temporarily affect the magnetic field.

Regardless the conservation of GZK-cutoff (Greisen, 1966; Zatsepin and Kuzmin, 1966), such a magnetic field would be able to accelerate the proton, for instance, up to $\sim 10^{22} \mathrm{eV}$. In this huge energy value, the well-known Fermi mechanisms are not taken into account.

So far, we conclude that the "grad E" drift (in timevarying magnetic field B) is very much powerful. It gives the largest energy value. Also, the polarization drift (in a time-varying electric field E) is able to raise the cosmic ray energy to an extreme value. It can be compared with the Hillas mechanism. The drift in a spatially-varying magnetic field $\mathrm{B}$ is almost as strong as the polarization drift. The fourth drift, the curvature a non-uniform magnetic field and a vanishing electric field, is very weak.

\section{REFERENCES}

Abraham, J., P. Abreubk, M. Agliettaav, C. Aguirrek and E.J. Ahn, et al., 2009. Upper limit on the cosmic-ray photon fraction at $\mathrm{EeV}$ energies from the pierre auger observatory. Astroparticle Phys., 31: 399-406. DOI: 10.1016/j.astropartphys.2009.04.003

Becker, W., 2009. X-Ray Emission from Pulsars and Neutron Stars. Neutr. Stars. Pulsars, 357: 91-140. DOI: 10.1007/978-3-540-76965-1_6
Bhattacharya, D. and C. Shukre, 1985. Has the Crab Pulsar Magnetic Field Grown after its Birth? J. Astrophys. Astr., 6: 233-237.

Blandford, R., J. Applegate and L. Hernquist, 1983. Thermal origin of neutron star magnetic fields. Mon. Not. Royal. Astr. Soc., 204: 1025-1048.

Blandford, R.D. and D. Eichler, 1987. Particle acceleration at astrophysical shocks: A theory of cosmic rays origin. Astrophys. J., 154: 175-175.

Collaboration, P.A., J. Abraham, P. Abreu, M. Aglietta, and C. Aguirre, 2007. Correlation of the highestenergy cosmic rays with nearby extragalactic objects. Science, 318: 938-43. PMID: 17991855

Contopoulos, J., D. Kazanas and, C. Fendt, 1999. The axisymmetric pulsar magnetosphere. Am. Astro Soc., 511: 351-358.

Fermi, E., 1949. On the origin of the cosmic radiation. Phys. Rev., 75: 1169.

Ghisellini, G., G. Ghirlanda, F. Tavecchio, F. Fraternali and G. Pareschi, 2008. Ultra-high energy cosmic rays, spiral galaxies and magnetars. Mon. Not. Royal Astr. Soc.: MNRAS, 390: L88-L92. DOI: 10.1111/j.1745-3933.2008.00547.x

Gopalswamy, N., S.S. Hasan and A. Ambastha, 2010. Heliophysical Processes. 1st Edn., Springer-Verlag, Heidel-Berg. ISBN: 978-3-642-11340-6, pp: 125.

Greisen, K., 1966. End to the cosmic-ray spectrum? Phys. Rev. Lett., 16: 748.

Hillas, A.M., 1984. The origin of ultra-high-energy cosmic rays. Ann. Rev. Astron. Astrophys., 22: 425444.

Inoue, S., 2008. Origin of ultra-high energy cosmic rays in the era of auger and telescope array. J. Phys. Conf. Ser., 120: 062001.

Nagano, M. and A. Watson, 2000. Observations and implications of the ultrahigh-energy cosmic rays. Rev. Mod. Phys. 72: 689-732.

Prescod-Weinstein, C., N. Afshordi and M.L. Balogh, 2009. Stellar black holes and the origin of cosmic acceleration. Phys. Rev. D., 80: 043513.

Price, D.J. and S. Rosswog, 2006. Producing ultrastrong magnetic fields in neutron star Mergers. Science, 312: 719-722. DOI: 10.1126/science. 1125201

Spruit, H.C., 2008. Magnetic fields and dynamics of the Sun's interior. Proceedings IAU Symposium, eds. K.G. Strassmeier, A.G. Kosovichev and J.E. Beckman, 259.

Takeda, M., N. Hayashida, K. Honda, N. Inoue and K. Kadota et al., 1998. Extension of the cosmic-ray energy spectrum beyond the predicted greisenZatsepin-Kuz'min Cutoff. Phys. Rev. Lett., 81: 1163-166. 
Tawfik, A., A. Saleh, M.T. Ghoneim and A.A. Hady, 2012. Acceleration and particle field interactions of cosmic rays I: Formalism.

Teshima, M., 2001. The highest energy cosmic rays. Prog. Theor. Phys. Suppl., 143: 114-124.

Woodward, J.F., 1984. Pulsar magnetogyro ratios and pulsar evolution. III-Magnetic field generation. Astrophys. J., 279: 803-806. Astrophys. J., 279: 803. 1984. DOI: $10.1086 / 161949$
Yoshida, S. and H. Dai, 1998. The extremely high energy cosmic rays. J. Phys., 24: 905-938.

Zatsepin, G. and V. Kuzmin, 1966. Upper limit of the spectrum of cosmic rays. J. Exp. Theor. Phys. Lett., 4: 78-80. 